ARTICLE

Received 8 Aug 2013 | Accepted 10 Jan 2014 | Published 30 Jan $2014 \quad$ DOl: 10.1038/ncomms4239

\title{
Polymorphism of dislocation core structures at the atomic scale
}

\author{
Zhongchang Wang ${ }^{1}$, Mitsuhiro Saito', Keith P. McKenna² \& Yuichi Ikuhara1,3,4,5
}

Dislocation defects together with their associated strain fields and segregated impurities are of considerable significance in many areas of materials science. However, their atomic-scale structures have remained extremely challenging to resolve, limiting our understanding of these ubiquitous defects. Here, by developing a complex modelling approach in combination with bicrystal experiments and systematic atomic-resolution imaging, we are now able to pinpoint individual dislocation cores at the atomic scale, leading to the discovery that even simple magnesium oxide can exhibit polymorphism of core structures for a given dislocation species. These polymorphic cores are associated with local variations in strain fields, segregation of defects, and electronic states, adding a new dimension to understanding the properties of dislocations in real materials. The findings advance our fundamental understanding of basic behaviours of dislocations and demonstrate that quantitative prediction and characterization of dislocations in real materials is possible.

\footnotetext{
${ }^{1}$ WPI, Advanced Institute for Materials Research, Tohoku University, 2-1-1 Katahira, Aoba-ku, Sendai 980-8577, Japan. ${ }^{2}$ Department of Physics, University of York, Heslington, York YO10 5DD, UK. ${ }^{3}$ Institute of Engineering Innovation, The University of Tokyo, 2-11-16, Yayoi, Bunkyo-ku, Tokyo 113-8656, Japan.

${ }^{4}$ Nanostructures Research Laboratory, Japan Fine Ceramics Center, 2-4-1 Mutsuno, Atsuta, Nagoya 456-8587, Japan. ${ }^{5}$ Center for Elements Strategy Initiative for Structure Materials, Kyoto University, Sakyo, Kyoto 606-8501, Japan. Correspondence and requests for materials should be addressed to Z.W. (email: zcwang@wpi-aimr.tohoku.ac.jp).
} 
$\mathrm{D}$ islocations, line defects in crystal lattices, and their interactions govern properties of many materials, ranging from fundamental plasticity and work hardening in metals and alloys to device pathology in semiconductor lightemitting and laser diodes or nonvolatile memories ${ }^{1-3}$. In addition, the elastic distortions of regular lattices around dislocations can give rise to a strain field with which the atomic impurities or point defects might interact, forming the so-called 'Cottrell atmosphere' that influences many areas of materials science $^{4}$. Technologically, dislocation lines can act as fast pathways for enhanced mass transport, an elemental phenomenon known as pipe diffusion, hence raising an appealing likelihood to synthesize low-dimensional hierarchical nanostructures in crystalline solids for dislocation-engineered devices,6. In all these circumstances, whether dislocations are vital or fatal, the atomic-scale structure of their cores, the locus where the lattice planes precisely terminate, assumes paramount importance.

Several experimental techniques exist for probing dislocations, but, in general, no one can individually provide sufficient information to constrain a systematic solution to their local core structure at the atomic scale ${ }^{7,8}$. X-ray and neutron scattering, as well as electron diffraction, yield a spatially averaged structure and are therefore insensitive to individual defects notwithstanding the increasingly high energy resolution. Although high-resolution atomic force microscopy enables individual dislocations to be identified at the surfaces, it falls short of providing atomic resolution of their cores ${ }^{9}$, impeding a ready inference of their core properties. In contrast, advanced transmission electron microscopy (TEM) is in principle able to directly resolve individual embedded dislocation cores with single-atom sensitivity ${ }^{10-13}$, particularly in the annular darkfield mode ${ }^{10,11}$. This leads to a far greater understanding on the correlation between the dislocation cores in real materials and their mechanical, optical, and electronic behaviours ${ }^{12,14}$.

Such an understanding is, however, incomplete in that the inherently local TEM imaging can, at best, reveal only a selection of dislocations in real materials, the nature of which is dictated by chemical composition, processing history (for example, mechanical deformation) and geometry of the sample (for example, lattice mismatched thin-film heterostructures) $)^{15}$. These substantially complicate our efforts to isolate and hence systematically pinpoint dislocations, thereby posing a relevant yet long unresolved problem in the field of materials science on whether dislocations of an individual type (defined by their characteristic displacement vectors, the Burgers vector $\mathbf{b}$ and line vector $\mathbf{t}$ ) can take on multiple core structures in real materials at the atomic scale. Addressing this issue has now become likely with the bicrystal technique that allows a precise control over crystallography ${ }^{16-18}$, while diffusion bonding two single crystals to introduce dislocations of a given type. However, the number of possible stable dislocation cores and their corresponding atomicscale structures are not known in advance. A coherent strategy is therefore indispensable to combine the experimental imaging with comprehensive theoretical simulations in a self-consistent global optimization scheme ${ }^{19,20}$.

Here we develop such a useful modelling strategy to identify atomic-scale geometries of all dislocation core polymorphs for a prescribed dislocation species (that is, $\mathbf{b}$ and $\mathbf{t}$ ) in $\mathrm{MgO}$, a prototypical ionic material that finds numerous technological applications where its prevalent dislocations are known or suspected to influence functionality, for example, the tunnel magnetoresistance effect in magnetic tunnel junctions ${ }^{21}$. We demonstrate the power and efficiency of such modelling approach by fabricating $\mathrm{MgO}$ bicrystals and directly imaging all predicted dislocation cores with sub-Ångström resolution, providing thereby definitive evidence for the polymorphism of dislocation cores at the atomic scale. By correlating element-selective imaging with the geometrical phase analysis (GPA) and quantummechanical calculations, we are able to identify distinct strain fields and local electronic states for the different dislocation cores, which shall have implications for the understanding of many properties and bring a novel dimension to dislocation-engineered materials.

\section{Results}

Theoretical prediction of dislocation core at the atomic scale. Inspired by the bicrystal concept, we build up a periodic array of one-dimensional edge dislocations in $\mathrm{MgO}$ by 'adjoining' its two single crystals, which are somewhat tilted with respect to each other, while retaining symmetry (Fig. 1a). The edge dislocation is chosen because it interacts most strongly with point defects owing to its associated pressure field, which is the key to clarify malfunction mechanisms of many existing devices, while raising hopes for making new ones, for example, straight quantum wires and dots in solids. Such a paradigm for producing dislocations has the advantage of uniquely defining their $\mathbf{b}$ and $\mathbf{t}$ vectors by manipulating the relative orientations of the two crystals. For example, the crystal orientation in Fig. 1a enables us to harvest dislocations with $\mathbf{b}=a[100]$ and $\mathbf{t}=[110]$ ( $a$ is lattice constant of $\mathrm{MgO}$ ). Moreover, the separations between parallel dislocations that are formed at the crystal interface can be precisely adjusted by prescribing the crystal misorientation angle $(\theta)^{5,17}$.

To predict atomic-scale structure of dislocations in the bicrystal, we construct atomistic models of symmetric low-angle boundaries between two $10 \mathrm{~nm}$-thick $\mathrm{MgO}$ crystals in the direction normal to interface (Fig. 1a), and impose periodic boundary conditions parallel to interface. Interactions between ions are described by classical polarizable interatomic potentials ${ }^{22}$ that are very accurate and efficient for ionic materials, thereby allowing an exhaustive search for stable interfaces by minimizing total energies with respect to ion coordinates and translation of one grain relative to the other (Fig. 1a). Such a procedure in general yields a series of metastable boundary structures involving different dislocation cores, their relative stability of which can be accessed by comparing interface formation energy defined as $E_{\mathrm{f}}=\left(E(\right.$ bicrystal $\left.)-N_{\mathrm{MgO}} E_{\mathrm{coh}}\right) / A$, where $E$ (bicrystal) is the energy of the bicrystal; $E_{\text {coh }}$ is the cohesive energy of bulk $\mathrm{MgO} ; N_{\mathrm{MgO}}$ is the number of $\mathrm{MgO}$ units; and $A$ is the interface $\mathrm{area}^{23}$. For a range of uncovered low-formation energy bicrystals, we create three-dimensionally periodic supercells containing dislocation dipoles and perform a structural energy minimization using density functional theory (DFT) calculations (Fig. 1b). In virtue of finite barriers to dislocation motion, we are able to acquire stable dislocation structures, despite that mutual interaction between dislocations may lead to their eventual annihilation.

Validation of bicrystal modelling approach. To address specific cases, we consider first the $\mathbf{b}=a / 2[110]$ edge dislocation in the slip system of $\mathrm{MgO}$, which is the most stable and has been investigated previously, thereby offering a useful validation to our approach. Figure 2a shows a predicted structure of the $a / 2[110]$ dislocation core, which represents the only stable one by a systematic search. A mirror plane is preserved passing through the dislocation core area, and this core holds an inconsiderable excess volume, as also revealed in simulated high-angle annular darkfield (HAADF) scanning TEM (STEM) image using the predicted model (Fig. 2b). Importantly, this dislocation core contains a string of fivefold under-coordinated ions at the tip of extra halfplane of atoms, in contrast to the sixfold coordination in the bulk $\mathrm{MgO}$, resulting in a deformation of the octahedra close to the core and hence local property shift ${ }^{24}$. 


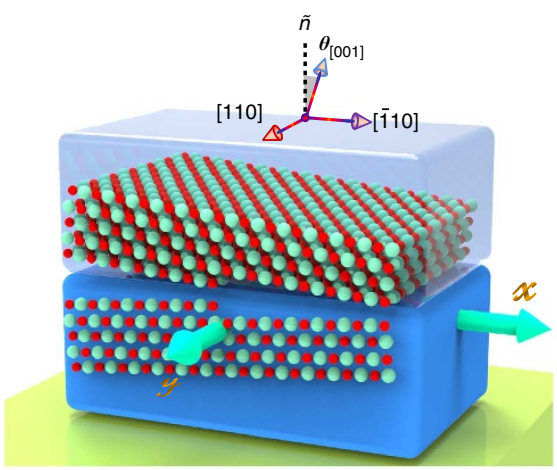

\section{b}

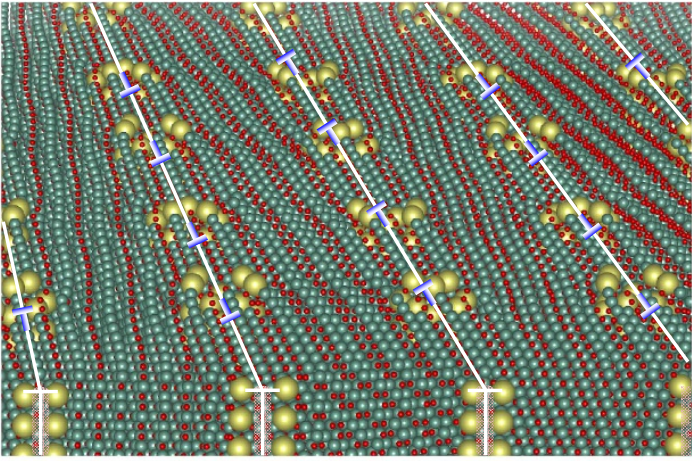

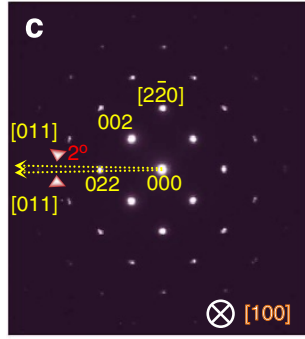
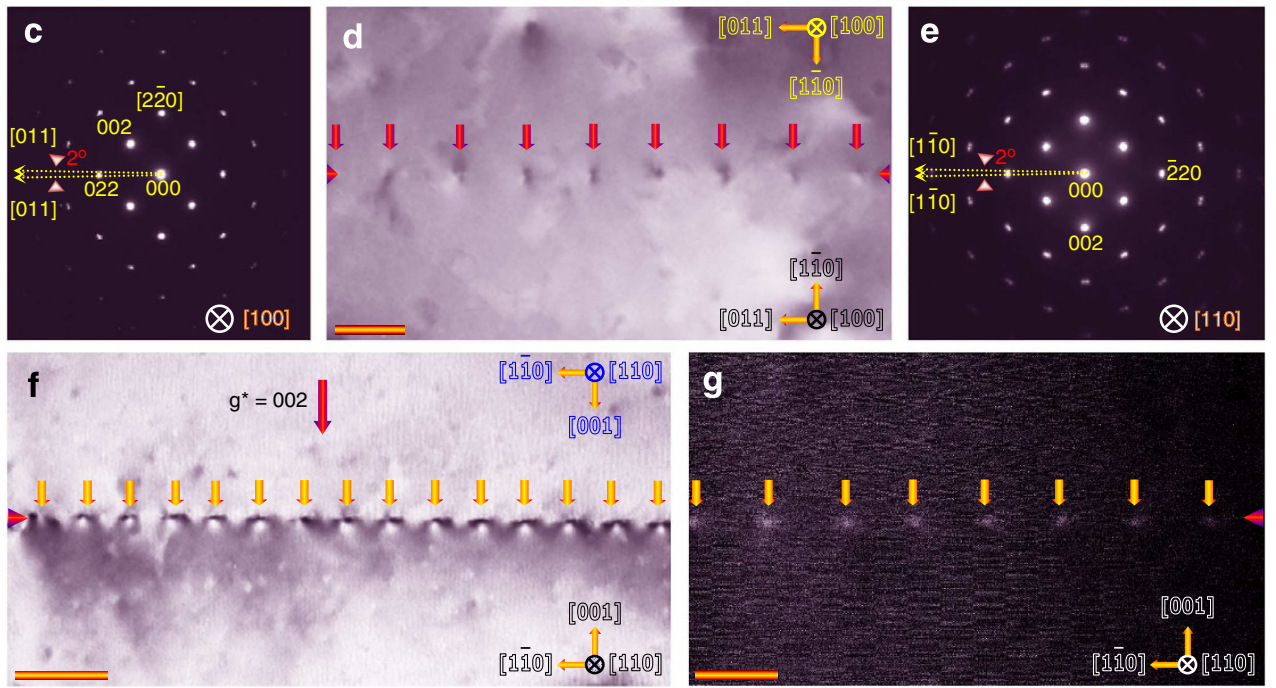

Figure 1 | Modelling and verification of dislocation array. (a) A one-dimensional edge dislocation array can be generated by adjoining two crystals misoriented about a common axis by a small angle $\theta$. To predict dislocation core structures, we performed an exhaustive search for all core configurations, taking into account the translation of one crystal with respect to the other (depicted by the arrows). The bigger balls in blue represent Mg and the smaller balls in red represent $\mathrm{O}$. (b) Three-dimensionally periodic supercells are constructed for the most stable dislocations and used to perform energy minimization using DFT. The atoms around dislocation cores are highlighted. (c) SADP is taken at the boundary of the (017)[100] bicrystal. (d) A representative cross-sectional bright-field TEM image of the boundary in the (01)[100] bicrystal, viewed along the [100] direction. A periodic image contrast is visible at the boundary area (indicated by arrows), suggesting the existence of embedded dislocation array. Scale bar, 10 nm.

(e) SADP taken at the boundary of the (001)[110] bicrystal. (f) A representative bright-field TEM image of the boundary in the (001)[110] bicrystal, viewed from the [110] direction. A periodic image contrast is unambiguously seen as well at the boundary area, inferring the presence of dislocation array. Scale bar, $20 \mathrm{~nm}$. (g) Low-magnification ADF STEM image of the boundary in the (001)[110] bicrystal, confirming the presence of a periodic one-dimensional dislocation array at the boundary. Scale bar, $10 \mathrm{~nm}$.

To test this predicted core structure, we fabricate a bicrystal with the bicrystallographic relations $(01 \overline{1})[100]_{\text {upper }} \|(01 \overline{1})$ $[100]_{\text {lower }}$ (Supplementary Fig. 1), that is, two crystals are sliced exactly along (01) plane of $\mathrm{MgO}$ lattice and the two finely polished surfaces are solid-state diffusion bonded with [100] direction of the upper crystal almost parallel to [100] direction of the lower one by a small tilt angle of $\theta=1^{\circ}$. Analysis of selected area diffraction pattern (SADP) taken at the boundary confirms these orientations to within the prescribed misfit tilt angle of $\sim 0.84^{\circ}$ estimated upon the splitting spots in the pattern (Fig. 1c), and the TEM characterization reveals a perfect joining with dislocations periodically aligned along the boundary (indicated by arrows in Fig. 1d). Further comprehensive $\mathbf{g}^{\star} \bullet \mathbf{b}$ analysis $\left(\mathbf{g}^{\star}\right.$ is the diffracting reciprocal lattice vector) of the dark-field images taken at a series of imaging conditions pinpoints that these dislocations are of edge type with $\mathbf{b}=a / 2[110]$ (Supplementary Fig. 2a,c). Upon closer inspection using Cs-corrected HAADF and annular bright-field $(\mathrm{ABF})^{25}$ STEM, as well as high-resolution TEM (HRTEM), we obtain a direct structural imaging of dislocation cores at atomic resolution, albeit the fact that their accompanying strain fields may remarkably blur lattice images. The bright spots in the HAADF STEM image represent the normal $\mathrm{MgO}$ atomic columns (Fig. 2c), unravelling a core geometry that is in line with the simulated HAADF STEM image using the predicted atomic model (Fig. 2b). Such a configuration is further verified in the ABF STEM (Supplementary Fig. 3b,c) and HRTEM images (Fig. 2d) in which the comprehensive image simulation recognizes the bright spots as sites of atomic columns (Supplementary Fig. 4a). We also conduct an atom-resolved imaging of the dislocations at many other boundary regions in several TEM specimens, and find interestingly that all acquired images point to identical core geometry, thereby providing a solid validation of our method.

Polymorphism of dislocation core structures. In addition to the a/2[110] dislocation, we extend the developed modelling strategy to systematically search core geometries of the $\mathbf{b}=a[100]$ edge dislocation, which has been discovered in $\mathrm{MgO}$ subjected to high-temperature electricfield treatment ${ }^{26}$. This dislocation has long been deemed as a culprit for catastrophic failure of the 

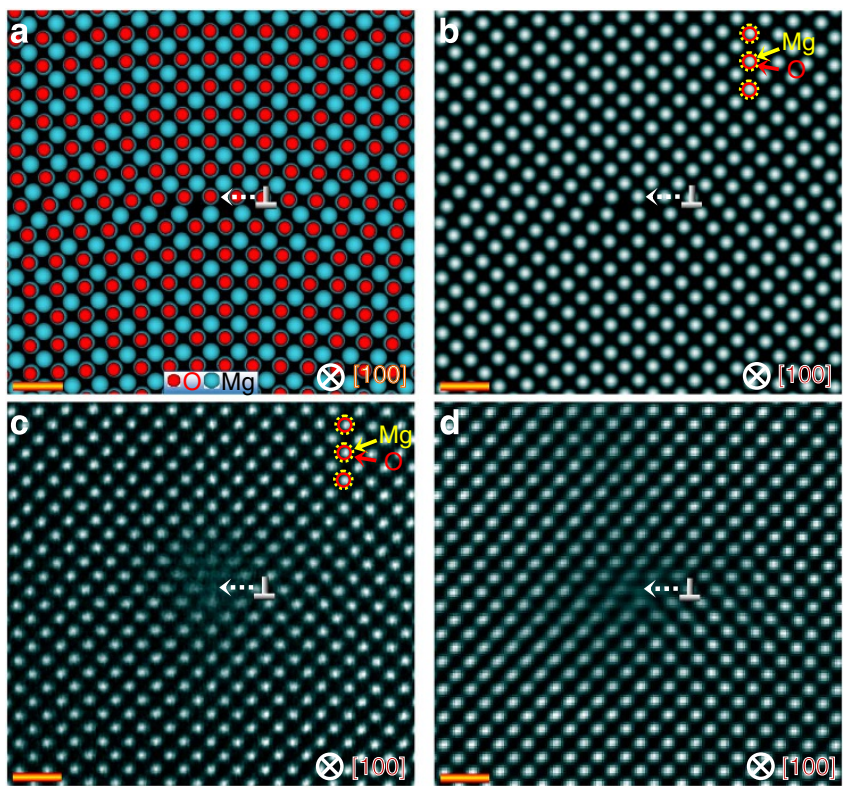

Figure 2 | Sole type of dislocation core in the slip system.

(a) Predicted model of an edge dislocation with $\mathbf{b}=a / 2$ [110], viewed from [100] direction. The arrows indicate the core location. Note that a systematic modelling uncovers only one dislocation core species in the primary slip system. (b) Simulated HAADF STEM image using the predicted core model. The dotted circle in yellow indicates $\mathrm{Mg}$ column and the dotted one in red indicates $\mathrm{O}$ column. The $\mathrm{Mg}$ and $\mathrm{O}$ columns are overlapped from [100] direction. (c,d) Atomic-resolution HAADF STEM (c) and HRTEM (d) images taken from [100] direction, which match the predicted model and associated simulated images well. It is noteworthy that many regions in several TEM samples have been explored and only one dislocation core type has been found. Scale bar, $5 \AA$.

intrinsically insulating properties of $\mathrm{MgO}$, yet a clear understanding of its thermal-electric breakdown still poses crucial challenges owing to the lack of atomistic detail of its cores. Based upon an exhaustive theoretical search by taking into account full ion optimization, translations of one crystal with respect to the other and addition and removal of ions at interface, as well as by calculating their associated formation energies, we predict three species of $a[100]$ dislocation core configurations (Fig. 3a-c) with similar formation energies $\left(1.7-2.4 \mathrm{~J} \mathrm{~m}^{-2}\right)$, in contrast to the sole type found for the $a / 2[110]$ dislocation $\left(0.89 \mathrm{~J} \mathrm{~m}^{-2}\right)$. The dislocation core that is predicted to be most stable, that is, having lowest $E_{\mathrm{f}}$ of $1.71 \mathrm{~J} \mathrm{~m}^{-2}$ (named type I), consists of a fairly open and symmetric core $\mathrm{e}^{27}$, and remains stable with respect to the insertion of additional atoms in the void (Fig. 3a), for example, adding four extra atomic columns raises $E_{\mathrm{f}}$ to $1.97 \mathrm{~J} \mathrm{~m}^{-2}$. The somewhat less stable dislocation core with a $E_{\mathrm{f}}$ of $2.13 \mathrm{~J} \mathrm{~m}^{-2}$ (named type II) contains a pair of climb partial dislocations (that is, bearing less than a unit lattice translation vector) bounding a stacking fault of $\sim 1.5 \mathrm{~nm}$ (cores are indicated by the arrows in Fig. 3b). In fact, we find interestingly a series of stable type II dislocations, which display varying spacings between the two partials, while imposing little impact on their formation energies. In contrast to the type I dislocation, the dislocation core with highest $E_{\mathrm{f}}$ of $2.39 \mathrm{~J} \mathrm{~m}^{-2}$ (named type III) turns more compact and hence induces heavier strain in the surrounding lattice (Fig. 3c). Structurally, it is considered as resulting from the two partial dislocations close together until ultimately a spatial symmetry is formed.

To verify the prediction that the a[100] dislocations show polymorphism at the atomic scale, we again rely on the geometry-tunable bicrystal technique to prepare boundaries with bicrystallographic relations $(001)[110]_{\text {upper }} \|(001)[110]_{\text {lower }}$ (Supplementary Fig. 1). The pre-designated orientations with a small misfit tilt angle of $\theta=\sim 1^{\circ}$ are testified in the diffraction pattern (Fig. 1e), ensuring a successful introduction of an array of dislocations aligned periodically at boundary, as seen in the TEM and STEM images (Fig. 1f,g). Upon a comprehensive $\mathbf{g}^{\star} \bullet \mathbf{b}$ analysis, these dislocations are pinpointed as edge ones with an identical Burgers vector $\mathbf{b}=a[100]$ (Supplementary Fig. 2). Further atomic-resolution imaging of individual dislocation at many boundary areas leads to a surprising conclusion that all of the probed dislocations eventually fall into three categories as revealed in the HAADF STEM images (Fig. 3d-f). The brighter spots in the HAADF images represent Mg columns, whereas lighter ones represent $\mathrm{O}$ columns, outlining the three core configurations that match elegantly with their predicted atomic models and simulated HAADF images (Fig. $3 g-i$ ). To add further support, we also acquire the atomic-resolution ABF STEM and HRTEM (Supplementary Figs 3 and 5) images for the three predicted dislocation cores, in good accord with their simulated counterparts using the three predicted models (Supplementary Fig. $4 \mathrm{~b}-\mathrm{d}$ ), thereby providing unequivocal evidence that various core polymorphs do exist for even a single-dislocation species at the atomic scale. The power and efficiency of such a complex modelling strategy can be further demonstrated by a direct atomresolved imaging of a complementary type II dislocation core involving the two partials separated further apart (Supplementary Fig. 6), in strong support of the theoretical predictions.

\section{Discussion}

For over five decades, TEM has enabled individual dislocations to be imaged, resulting in a much improved understanding of the interplay between defect structures of materials and their properties. Some of the traditional views, however, may need to be reconsidered, as the resulting implications rest more on a selective imaging than on a comprehensive atomic characterization. Such calling for a reinterpretation of existing structure-property relationships should now become particularly imminent because the current discovery of core polymorphism at the atomic scale adds instantly a novel dimension to the established characterization scheme that highlights the effect of dislocations with different $\mathbf{b}$ and $\mathbf{t}$ vector $^{28}$. It should be noted that for very low-angle grain boundaries where dislocations are separated well enough to be considered isolated, that is, dislocation cores are surrounded by regions of perfect crystal, such dislocation core polymorphism cannot be simply represented by structural multiplicity of grain boundaries (Supplementary Discussion). This first (to our knowledge) finding in $\mathrm{MgO}$ also takes on practical significance in nanoelectronic devices where miniaturization immediately requires a priori knowledge of dislocation core structures in that they are often associated with notably different strain fields, tendencies to accumulate defects or impurities and local electronic states, all of which dominate device functionalities.

To quantify local differences and shed light on the consequence of core polymorphism, we first perform a GPA of the aberrationcorrected HRTEM images to assess strain fields associated with the dislocations ${ }^{29}$, a quantity responsible for many phenomena in material science. The aberration corrector is harnessed to suppress substantially lattice distortions in a conventional HRTEM image arising from projection lens, thereby allowing a precise displacement measurement at the atomic level. The strain field $\left(\varepsilon_{\mathrm{xx}}\right)$ is spatially localized $(\sim 3 \mathrm{~nm})$ and relies heavily on specific dislocation cores (Fig. $4 \mathrm{a}-\mathrm{d}$ ), underscoring the relevance in unravelling all cores for one dislocation type at the atomic scale. It is noteworthy that our calculated dislocation interval 

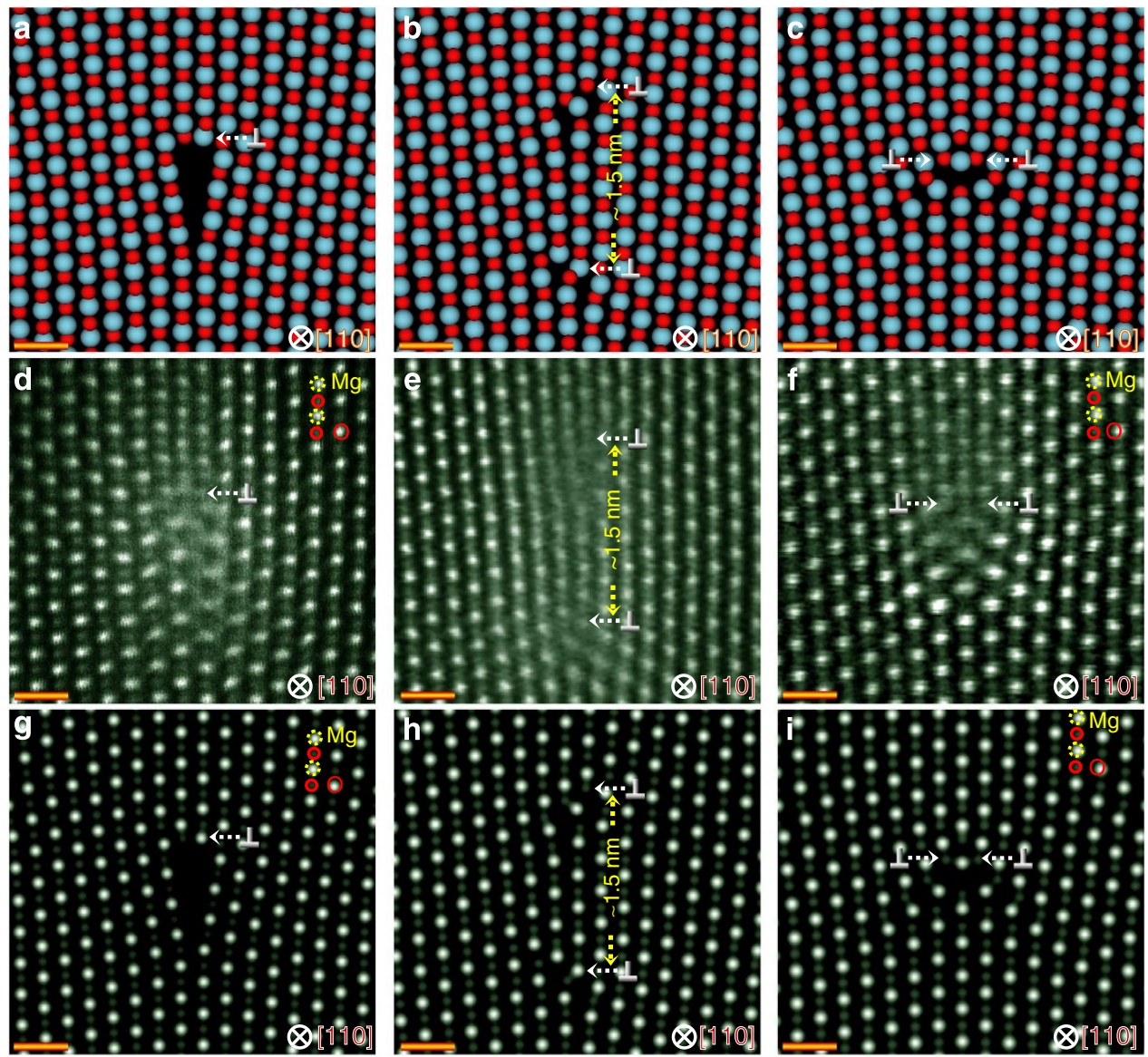

Figure 3 | Polymorphism of dislocation cores at the atomic scale. (a-c), Three models are predicted for the $a[100]$ dislocations: a single $\mathbf{b}=a[100]$ dislocation with no splitting (type I) (a); two $\mathbf{b}=a / 2$ [100] partial dislocations bounding a stacking fault of $\sim 1.5 \mathrm{~nm}$ (type II) (b); and a single $\mathbf{b}=a[100]$ dislocation with splitting (type III) (c). The three core structures differ locally from one another, albeit the fact that they share the same Burgers vector, thereby underscoring the importance in identifying each individual core structures for even a single dislocation. (d-f) HAADF STEM images of the type I (d), II (e), and III (f) dislocations taken from [110] direction, consistent with the predicted atomic models both geometrically and in quantity. The $\mathrm{Mg}$ columns (indicated by larger circles) are distinguishable from O columns (indicated by smaller circles) along the [110] direction. Special care has been taken with the imaging because the strain fields associated with the cores may blur the images. (g-i) Simulated HAADF STEM images of the $a[100]$ dislocations obtained using the predicted models: type I (g), II (h) and III (i) dislocations. Scale bar, $5 \AA$.

$(\sim 12 \mathrm{~nm})$ is much longer than the characteristic radius of the associated dislocation strain field $(\sim 3 \mathrm{~nm})$ indicating that the dislocations are separated to such a degree that they can be viewed isolated. Dislocations in our very low-angle grain boundaries can hence be considered to represent bulk lattice dislocations and have an equivalent core structure (Supplementary Discussion). Interestingly, the $a / 2[110]$ and type III $a[100]$ dislocations can give rise to a typical compression-tension strain field pair at their cores, whereas the others display an extra converse butterfly-tie-like pair region in between, which may provide varying driving force for impurity segregation towards dislocations. This scenario can be verified in the summed electron energy-loss spectra acquired from both the bulk and core area showing segregation of $\mathrm{Ca}$ and $\mathrm{Ti}$ to the three otherwise impurity-free $a[100]$ dislocations, yet no impurity segregation at all to the $a / 2[110]$ dislocation (Supplementary Fig. 7). The presence of $\mathrm{Ca}$ at the cores is not surprising, given that it is the majority impurity in $\mathrm{MgO}$ (ref. 30), is isovalent, and exhibits a strong segregation tendency owing to the ion-size misfit effect. The $\mathrm{Ti}$ impurity, however, may be either introduced unintentionally during the surface treatments of the $\mathrm{MgO}$ single crystals and fabrication of the bicrystals or segregated from the $\mathrm{MgO}$ bulk, although it is not detectable in the bulk. Such presence of Ti could be because of the space charge of the dislocation cores or as a result of either thermodynamics (that is, enhanced segregation) or kinetics (that is, enhanced diffusion) effect. The different ability to trap impurities or defects may account for the thermal-electric breakdown in $\mathrm{MgO}$ sample where a large amount of $a[100]$ dislocations are formed 26

Figure $4 \mathrm{e}-\mathrm{g}$ shows two-dimensional electron energy-loss spectra maps of the $\mathrm{Ca}-\mathrm{L}_{2,3}, \mathrm{Ti}-\mathrm{L}_{2,3}$ and $\mathrm{O}-\mathrm{K}$ edges by focusing on the core area of $a[100]$ dislocations. Ca prefers to segregate to the dilated part of edge dislocation in that it is oversized in $\mathrm{Mg}$ substitution, while size-comparable Ti resides at either compressive or tensile region depending on the specific core type and segregation circumstance ${ }^{30}$. Oxygen is, however, uniformly distributed regardless of the dislocation core type. Apart from the different spatial segregation behaviour, the multiple cores are accompanied by characteristic discrepancies in electronic properties as well, as our DFT calculations reveal qualitatively new gap states for all the four dislocation cores even in the absence of impurities, which vary with dislocation core types (Supplementary Fig. 8). These gap states clearly originate from the defective structures at the dislocation core, that is, both under-coordination of ions and local strain. Further analysis of interatomic bond length at the core reveals that it varies substantially at the core area, and the degree of variation depends heavily on specific core species as well (Supplementary 

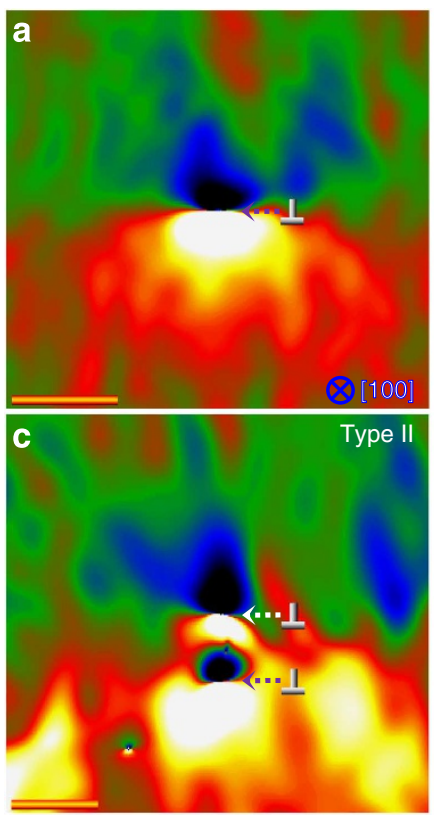
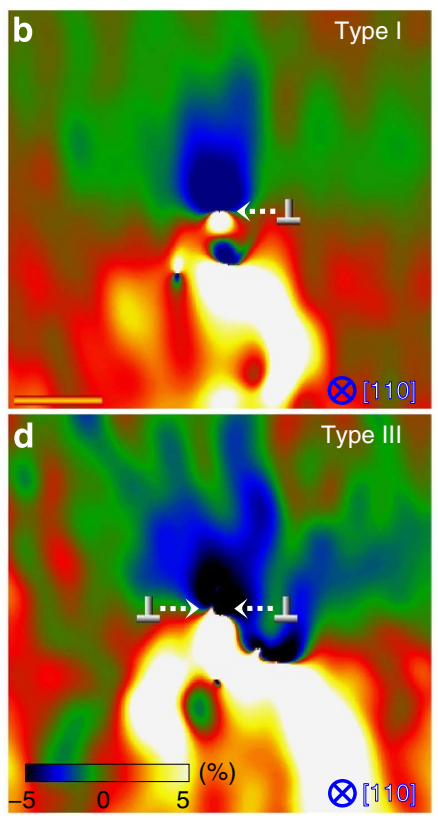
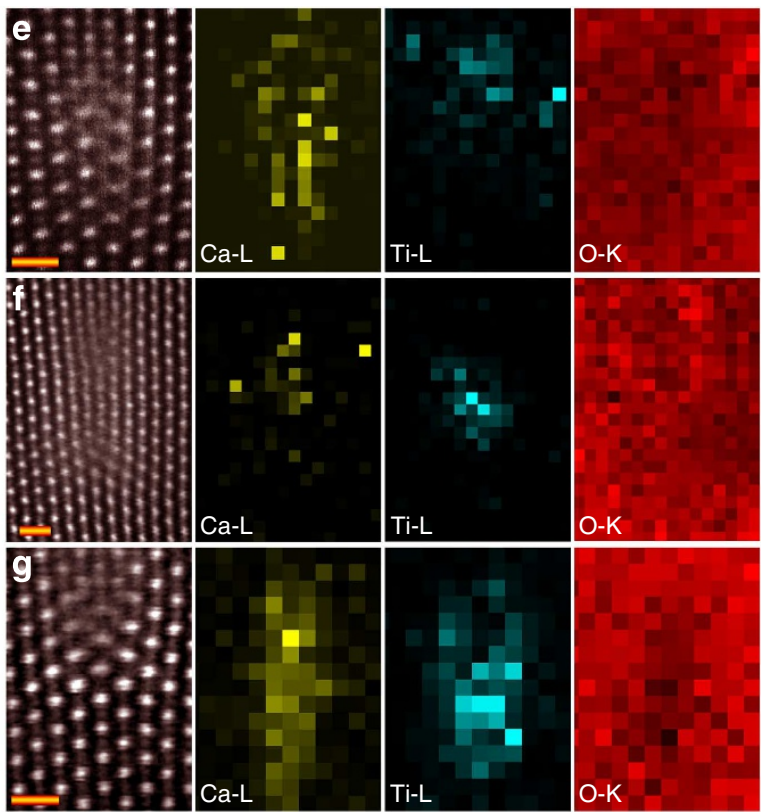

Figure 4 | Local property shift for polymorphic dislocation cores. (a-d) Strain maps of the in-plane $\varepsilon_{\mathrm{xx}}$ calculated by the GPA for the $a / 2[110]$ (a) and the type I (b), II (c) and III (d) a[100] dislocations. The GPA is performed using the obtained aberration-corrected HRTEM image. The $x$ and $y$ directions are predefined as [011] and [011] for the $a / 2$ [110] dislocation, whereas as [001] and [110] for the $a$ [100] dislocations, respectively. The colour bar indicates change in strain intensity from $-5.0 \%$ (compressive) to 5.0\% (tensile). Scale bar, 2 nm. (e-g) Magnified HAADF STEM images and core-loss images of the Ca- $\mathrm{L}_{2,3}, \mathrm{Ti}-\mathrm{L}_{2,3}$ and $\mathrm{O}-\mathrm{K}$ edges viewed from [110] direction for the type I (e), II (f) and III (g) a[100] dislocations. Core-loss images are made where the HAADF STEM images are taken. Scale bar, $5 \AA$.

Fig. 9a-d). However, the interatomic bonding at the core maintains ionic nature as in the case of bulk $\mathrm{MgO}$, irrespective of the different core species, as shown in the isosurface plots of charge density (Supplementary Fig. 9e-h).

The close match of predicted dislocation cores with direct imaging both geometrically and in quantity offers confidence that the modelling approach represents a reliable routine to pinpointing all core configurations for even a given dislocation type at the atomic scale. Such ability should afford new perspectives on dislocation activities that have hitherto been explored either selectively or in average, providing therefore a basis for inferring the dislocation-related mechanisms and for designing the dislocation-engineered devices. The finding that dislocation cores can exhibit multiple structures and local property variations promises to advance our understanding on properties of $\mathrm{MgO}$ including the abnormal rise in electrical conductivity. Such a modelling paradigm that allows atomic-scale resolving of dislocation core polymorphism is general and should be applicable to many complex materials in which the behaviour of dislocation cores is decisive to understand a range of properties.

\section{Methods}

Calculational process and methodology. The dislocation core structures were predicted using the theoretical bicrystal approach implemented in the METADISE $\operatorname{code}^{31}$. It involved constructing a two-dimensionally periodic interface between two grains that were oriented at any prescribed angles (Supplementary Fig. 1a). The grains were modelled with periodic boundary conditions parallel to interface and were sufficiently wide along interface normal, such that relevant properties and interface formation energies were converged. In the calculations presented here, each crystal was as thick as $10 \mathrm{~nm}$ in the direction normal to interface. Interactions between ions in the bicrystal were described by polarizable interatomic potentials ${ }^{22}$ that have been shown to be accurate and efficient for many ionic materials. Ions within $4 \mathrm{~nm}$ of the interface were allowed to relax, whereas more distant ions were held fixed at their bulk sites. It is noteworthy that this approach is in principle not limited to such potentials. Recent advances in the charge equilibration potentials $^{32,33}$ and linear-scaling DFT ${ }^{34}$ could open the way to applying this modelling approach to more complex crystals including impurity segregation.
Structures of the bicrystals were fully optimized with respect to the positions of ions near the interface, rigid translation of one crystal with respect to the other, and addition and removal of ions at the interface. Such a procedure in general yielded a series of boundary structures involving different dislocation cores, the relative stability of which could be assessed by comparing interface formation energy defined as $E_{\mathrm{f}}=\left(E\right.$ (bicrystal) $\left.-N_{\mathrm{MgO}} E_{\text {coh }}\right) / A$, where $E$ (bicrystal) was the energy of bicrystal; $E_{\mathrm{coh}}$ was the cohesive energy of bulk $\mathrm{MgO} ; N_{\mathrm{MgO}}$ was the number of $\mathrm{MgO}$ units; and $A$ was the interface area. For the most stable inequivalent bicrystal structures, we had created three-dimensionally periodic supercells containing two dislocation dipoles, which were fully optimized using the GULP code.

DFT calculation. The supercells served as initial models for further DFT calculations using Vienna $a b$ initio simulation package ${ }^{35}$. We applied the projector augmented-wave method ${ }^{36}$ with $4 \times 1 \times 1 k$-point grids and a cutoff energy of $400 \mathrm{eV}$, which enabled an accurate prediction of both atomic and electronic structures of the dislocation systems. Dislocation cores were modelled with large periodic supercells containing up to 1,600 atoms using the DFT, which were adopted further for the image simulations. All atoms in the supercells were fully optimized until the magnitude of force on every atom converged to $<0.05 \mathrm{eV}$ per $\AA$, yielding optimized structures. It is worth mentioning that DFT calculations retain the dislocation core structures predicted by the classical polarizable interatomic potentials, thereby validating the application of these potentials to predict dislocation cores in ionic oxide $\mathrm{MgO}$. In addition, it should be noted that electronic structures of every dislocation core have been calculated using the DFT in order to reveal the electronic difference between the discovered dislocation cores.

Bicrystal sample fabrication. Two classes of $\mathrm{MgO}$ bicrystals with a small tilt angle of $\theta=1^{\circ}$ were prepared by the bicrystal technique by joining two pristine singlecrystal blocks (upper and lower) of MgO (purity: 99.9\%, Shinkosha, Tokyo): one had the bicrystallographic relationships of $(01 \overline{1})[100]_{\text {upper }} \|(01 \overline{1})[100]_{\text {lower }}$ and the other $(001)[110]_{\text {upper }} \|(001)[110]_{\text {lower, }}$ as sketched in Supplementary Fig. 1a. The size of each single-crystal block was set to be $10 \times 10 \times 5 \mathrm{~mm}^{3}$ and thus $10-\mathrm{mm}$ cubic bicrystals were produced. To fabricate the predesigned bicrystals with the confined dislocation arrays, the two single crystals were first cut precisely along $(01 \overline{1})$ (or (001)) plane of MgO lattices, followed by mechanical grinding and polishing of the two contacting planes to mirror finish with diamond slurry of $0.25 \mu \mathrm{m}$. Next, the two revealed surfaces were bonded face to face with the [100] (or [110]) direction of one crystal almost parallel to the [100] (or [110]) direction of the other by the small tilt angle of $\sim 1^{\circ}$ under hot pressing at $1,773 \mathrm{~K}$ for $10 \mathrm{~h}$ in air. The picture of final bicrystal was given in Supplementary Fig. 1b, in which dislocations were expected to lie at the boundary region indicated by arrows. 
The heating and cooling rates were set to be $300 \mathrm{Kh}^{-1}$ during pressing. Several slices with a dimension of $10 \times 10 \times 1.5 \mathrm{~mm}^{3}$ were cut from the final bicrystal blocks to prepare specimens for TEM and STEM observations.

TEM specimen preparation and imaging condition. Thin-foil TEM and STEM specimens were prepared by first cutting slices from the final bicrystal blocks along (011) (or (110)) plane. The slices were then mechanically ground till their thickness reached $\sim 0.12 \mathrm{~mm}$, which was followed by a further dimpling down to $\sim 20 \mu \mathrm{m}$. To make electron transparent, the dimpled slices were finally thinned by argon-ion sputter beam using PIPS 691 (Gatan Co., Ltd.). A gun voltage of $1-4 \mathrm{kV}$ and an incident beam angle of $4-6^{\circ}$ were used in order to avoid, to maximum extent, radiation damage to the sample. SADPs, TEM and HRTEM images were taken using the JEOL JEM-2010F and FEI TITAN80-300 electron microscopes equipped with image corrector. The HAADF and ABF images were taken with the 200-kV JEM$2100 \mathrm{~F}$ and ARM-200FC STEM, both of which were equipped with a probe corrector (CEOS Gmbh), providing thereby unprecedented opportunity to probe structures with sub-Ångström resolution. For the HAADF STEM imaging, a probe convergence angle of $\sim 22 \mathrm{mrad}$ and a detector with an inner semi-angle of over $60 \mathrm{mrad}$ were adopted. ABF STEM images were made with a 6-25 mrad detector, and electron energy-loss spectra was recorded using a Gatan Enfina system equipped on the STEM with an energy resolution, at full-width of half-maximum, of $\sim 0.5 \mathrm{eV}$.

Image simulation and GPA. Both HRTEM and HAADF STEM image simulations were performed using the commercially available WinHREM package (HREM Research Inc.), which was based upon the multislice method. The fully optimized atomic models obtained from the DFT calculations were adopted for image simulations. The spherical and chromatic aberration coefficients were assumed as $C_{\mathrm{s}}=0.7 \mathrm{~mm}$ and $C_{\mathrm{c}}=1.6 \mathrm{~mm}$, respectively, to simulate the HRTEM images. The $200-\mathrm{kV}$ probe was assumed to have a probe-forming aperture of $22 \mathrm{mrad}$ and the ADF annular detector was adopted to span the range of $81-228 \mathrm{mrad}$. Debye-Waller factor was taken into account for each element involved, yet was averaged over spatial directions, namely, the anisotropy of the absorption of thermal diffuse scattering factors was ignored in the image simulations. The $\mathrm{GPA}^{29,37}$ was performed based on the aberration-corrected HRTEM images using a GPA plug-in package (HREM Research Inc.), which was implemented in the Digital Micrograph software (Gatan).

\section{References}

1. Ma, E., Shen, T. D. \& Wu, X. L. Nanostructured metals: Less is more. Nat. Mater. 5, 515-516 (2006).

2. Nakamura, S. The roles of structural imperfections in InGaN-based blue light-emitting diodes and laser diodes. Science 281, 956-961 (1998).

3. Sugiyama, I. et al. Ferromagnetic dislocations in antiferromagnetic NiO. Nat. Nanotechnol. 8, 266-270 (2013).

4. Blavette, D., Cadel, E., Fraczkiewicz, A. \& Menand, A. Three-dimensional atomic-scale imaging of impurity segregation to line defects. Science 286, 2317-2319 (1999).

5. Ikuhara, Y. Nanowire design by dislocation technology. Prog. Mater. Sci. 54, 770-791 (2009).

6. Nakamura, A., Matsunaga, K., Tohma, J., Yamamoto, T. \& Ikuhara, Y. Conducting nanowires in insulating ceramics. Nat. Mater. 2, 453-456 (2003).

7. Billinge, S. J. L. \& Levin, I. The problem with determining atomic structure at the nanoscale. Science 316, 561-565 (2007).

8. Barnard, J. S., Sharp, J., Tong, J. R. \& Midgley, P. A. High-resolution three-dimensional imaging of dislocations. Science 313, 319 (2006).

9. Moram, M. A., Oliver, R. A., Kappers, M. J. \& Humphreys, C. J. The spatial distribution of threading dislocations in gallium nitride films. Adv. Mater. 21, 3941-3944 (2009).

10. Shibata, N. et al. Nonstoichiometric dislocation cores in $\alpha$-alumina. Science 316, 82-85 (2007).

11. Muller, D. A. Structure and bonding at the atomic scale by scanning transmission electron microscopy. Nat. Mater. 8, 263-270 (2009).

12. Heuer, A. H., Jia, C. L. \& Lagerlöf, K. P. D. The core structure of basal dislocations in deformed sapphire $\left(\alpha-\mathrm{Al}_{2} \mathrm{O}_{3}\right)$. Science 330, 1227-1231 (2010).

13. Chen, C. et al. Three-dimensional imaging of dislocations in a nanoparticle at atomic resolution. Nature 496, 74-77 (2013).

14. Arslan, I. \& Bowning, N. D. Intrinsic electronic structure of threading dislocations in GaN. Phys. Rev. B 65, 075310 (2002).

15. Marks, R. A., Taylor, S., Mammana, T. E., Gronsky, R. \& Glaeser, A. M Directed assembly of controlled-misorientation bicrystals. Nat. Mater. 3, 682-686 (2004)

16. Duscher, G., Chisholm, M. F., Alber, U. \& Rühle, M. Bismuth-induced embrittlement of copper grain boundaries. Nat. Mater. 3, 621-626 (2004).

17. Ikuhara, Y. et al. Dislocation structures of low-angle and near- $\Sigma 3$ grain boundaries in alumina bicrystals. J. Am. Ceram. Soc. 86, 595-602 (2003).

18. Tochigi, E., Shibata, N., Nakamura, A., Yamamoto, T. \& Ikuhara, Y. Partial dislocation configurations in a low-angle boundary in $\alpha-\mathrm{Al}_{2} \mathrm{O}_{3}$. Acta Mater. 56, 2015-2021 (2008).
19. Woodward, C., Trinkle, D. R., Hector, Jr L. G. \& Olmsted, D. L. Prediction of dislocation cores in aluminum from density functional theory. Phys. Rev. Lett. 100, 045507 (2008)

20. Proville, L., Rodney, D. \& Marinica, M. Quantum effect on thermally activated glide of dislocations. Nat. Mater. 11, 845-849 (2012).

21. Ikeda, S. et al. A perpendicular-anisotropy CoFeB-MgO magnetic tunnel junction. Nat. Mater. 9, 721-724 (2010).

22. Lewis, G. V. \& Catlow, C. R. A. Potential models for ionic oxides. J. Phys. C 18, 1149-1161 (1985).

23. Chua, A. L.-S., Benedek, N. A., Chen, L., Finnis, M. W. \& Sutton, A. P. A genetic algorithm for predicting the structures of interfaces in multicomponent systems. Nat. Mater. 9, 418-422 (2010).

24. Zhang, F., Walker, A. M., Wright, K. J. \& Gale, D. Defects and dislocations in $\mathrm{MgO}$ : atomic scale models of impurity segregation and fast pipe diffusion. J. Mater. Chem. 20, 10445-10451 (2010).

25. Findlay, S. et al. Robust atomic resolution imaging of light elements using scanning transmission electron microscopy. Appl. Phys. Lett. 95, 191913 (2009)

26. Narayan, J., Weeks, R. A. \& Sonder, E. Aggregation of defects and thermalelectric breakdown in MgO. J. Appl. Phys. 49, 5977-5981 (1978).

27. McKenna, K. P. \& Shluger, A. L. Electron-trapping polycrystalline materials with negative electron affinity. Nat. Mater. 7, 859-862 (2008).

28. Lopatin, S., Pennycook, S. J., Narayan, J. \& Duscher, G. Z-contrast imaging of dislocation cores at the GaAs/Si interface. Appl. Phys. Lett. 81, 2728 (2002).

29. Hÿtch, M. J., Putaux, J.-L. \& Pénisson, J.-M. Measurement of the displacement field of dislocations to $0.03 \AA$ by electron microscopy. Nature 423, 270-273 (2003).

30. Wang, Z. C. et al. Atom-resolved imaging of ordered defect superstructures at individual grain boundaries. Nature 479, 380-383 (2011).

31. Watson, G. W., Kelsey, E. T., de Leeuw, N. H., Harris, J. D. \& Parker, S. C. Atomistic simulation of dislocations, surfaces and interfaces in MgO. J. Chem Soc. Faraday Trans. 92, 433-438 (1996).

32. van Duin, A. C. T. et al. ReaxFF $\mathrm{SiO}_{\mathrm{S}}$ reactive force field for silicon and silicon oxide systems. J. Phys. Chem. A 107, 3803-3811 (2003).

33. Shan, T.-R., Devine, B. D., Kemper, T. W., Sinnott, S. B. \& Phillpot, S. R. Charge optimized many-body potential for the hafnium/hafnium oxide system. Phy. Rev. B 81, 125328 (2010).

34. Skylaris, C.-K., Haynes, P. D., Mostofi, A. A. \& Payne, M. C. Introducing ONETEP: Linear-scaling density functional simulations on parallel computers. J. Chem. Phys. 122, 084119 (2005).

35. Kresse, G. \& Furthmuller, J. Efficient iterative schemes for $a b$ initio total-energy calculations using a plane-wave basis set. Phys. Rev. B 54, 11169-11186 (1996)

36. Blöchl, P. E. Projector augmented-wave method. Phys. Rev. B 50, 17953-17979 (1994).

37. Hÿtch, M. J., Snoeck, E. \& Kilaas, R. Quantitative measurement of displacement and strain fields from HREM micrographs. Ultramicroscopy 74, 131-146 (1998).

\section{Acknowledgements}

This work was conducted in part in the Research Hub for Advanced Nano Characterization and the 'Nanotechnology Platform' at the University of Tokyo supported by the MEXT of Japan, and was supported in part by the Elements Strategy Initiative for Structural Materials by the MEXT by Japan. Z.W. thanks the financial supports from the Grant-in-Aid for Young Scientists (A) (grant no. 24686069), the Challenging Exploratory Research (grant no. 24656376), the Sasakawa Scientific Research Grant and the JGC-S Foundation. K.P.M acknowledges the financial support from The Engineering and Physical Science Research Council (EPSRC) (grant EP/K003151) and access to highperformance computational resource by UK's Materials Chemistry Consortium (EPSRC grant EP/F067496). Calculations were conducted in part at the Institute for Solid State Physics (ISSP), University of Tokyo.

\section{Author contributions}

Z.W. prepared specimen, carried out calculations and wrote the paper. M.S. took images and conducted image processing. K.P.M. performed calculations. Y.I. discussed the results and directed the entire study. All authors read and commented on the paper.

\section{Additional information}

Supplementary Information accompanies this paper at http://www.nature.com/ naturecommunications

Competing financial interests: The authors declare no competing financial interests

Reprints and permission information is available online at http://npg.nature.com/ reprintsandpermissions/

How to cite this article: Wang, Z. et al. Polymorphism of dislocation core structures at the atomic scale. Nat. Commun. 5:3239 doi: 10.1038/ncomms4239 (2014). 